\title{
Correction to: Knowledge of psychosocial factors associated with low back pain amongst health science students: a scoping review
}

Kelsey L. Lewis ${ }^{*}$ and Patrick J. Battaglia

\section{Correction to: Chiropr Man Ther (2019) 27:64 \\ https://doi.org/10.1186/s12998-019-0284-5}

Following publication of the original article [1], we have been notified that one of the author names was incorrect.

Originally published author name:

- Patrick Battglia

Correct author name:

- Patrick Battaglia

The original article has been corrected.

Published online: 26 November 2019

\section{Reference}

1. Lewis KL, Battaglia PJ. Knowledge of psychosocial factors associated with low back pain amongst health science students: a scoping review. Chiropr Man Ther. 2019;27:64. https://doi.org/10.1186/s12998-019-0284-5.

* Correspondence: Kelsey.lewis@loganhealthcenters.com 